\title{
Correction to: Inner child of the past: long-term protective role of childhood relationships with mothers and fathers and maternal support for mental health in middle and late adulthood
}

\author{
Ping Chen ${ }^{1}$ (I)
}

Published online: 13 December 2021

This is a U.S. government work and not under copyright protection in the U.S.; foreign copyright protection may apply 2021

\section{Correction to: \\ Social Psychiatry and Psychiatric Epidemiology \\ https://doi.org/10.1007/s00127-021-02200-y}

In the original version of the article, in Table 1, the second heading cells for "Full sample Female Male" and the fourth heading cells for "Full sample Female Male" should be deleted and texts of "Fair", "Poor", "Mean", "86-90 years***" and "91-95 years***" should be indented to line up the text in the previous row.

The corrected table (Table 1) should look similar to the following:

The original article has been corrected.

The original article can be found online at https://doi.org/10.1007/ s00127-021-02200-y.

Ping Chen

pc@unc.edu

1 Frank Porter Graham Child Development Institute,

University of North Carolina at Chapel Hill, Campus Box

\#8180, Chapel Hill, NC 27599-8180, USA 
Table 1 Sample demographics and study characteristics

\begin{tabular}{|c|c|c|c|}
\hline & $\begin{array}{l}\text { Full sample } \\
(N=12,606)\end{array}$ & $\begin{array}{l}\text { Female } \\
(N=7,319)\end{array}$ & $\begin{array}{l}\text { Male } \\
(N=5,287)\end{array}$ \\
\hline \multicolumn{4}{|l|}{ Mother-child relationship before age $18, \mathrm{~N}$ (percent) } \\
\hline Poor & $2412(19.13)$ & $1663(22.72)$ & $749(14.17)$ \\
\hline Good & $10,194(80.87)$ & $5656(77.28)$ & $4538(85.83)$ \\
\hline \multicolumn{4}{|l|}{ Father-child relationship before age $18, \mathrm{~N}$ (percent) } \\
\hline Poor & $3230(25.62)$ & $1926(26.32)$ & $1304(24.66)$ \\
\hline Good & $9376(74.38)$ & $5393(73.68)$ & $3983(75.34)$ \\
\hline Mother's attention and time (range $1-4$ ), mean (SD) & $3.49(0.0068)$ & $3.44(0.0094)$ & $3.56(0.0098)$ \\
\hline Mother's effort watching over (range $1-4$ ), mean (SD) & $3.64(0.0062)$ & $3.62(0.0084)$ & $3.67(0.0090)$ \\
\hline \multicolumn{4}{|l|}{ Age group } \\
\hline $51-55$ years & $2073(16.44)$ & $1237(16.9)$ & $836(15.81)$ \\
\hline $56-60$ years & $2436(19.32)$ & $1422(19.43)$ & $1014(19.18)$ \\
\hline $61-65$ years & $1589(12.61)$ & $982(13.42)$ & $607(11.48)$ \\
\hline $66-70$ years & $2259(17.92)$ & $1278(17.46)$ & $981(18.55)$ \\
\hline $71-75$ years & $1832(14.53)$ & $1010(13.8)$ & $822(15.55)$ \\
\hline $76-80$ years & $1173(9.31)$ & $656(8.96)$ & $517(9.78)$ \\
\hline $81-85$ years & $739(5.86)$ & $415(5.67)$ & $324(6.13)$ \\
\hline $86-90$ years & $396(3.14)$ & $252(3.44)$ & $144(2.72)$ \\
\hline 91-95 years & $109(0.86)$ & $67(0.92)$ & $42(0.79)$ \\
\hline \multicolumn{4}{|l|}{ Race/ethnicity, $N$ (percent) } \\
\hline Non-Hispanic white & $9646(76.52)$ & $5517(75.38)$ & $4129(78.1)$ \\
\hline Non-Hispanic black & $1530(12.14)$ & $978(13.36)$ & $552(10.44)$ \\
\hline Non-Hispanic other race & $315(2.5)$ & $177(2.42)$ & $138(2.61)$ \\
\hline Hispanic & $1115(8.84)$ & $647(8.84)$ & $468(8.85)$ \\
\hline \multicolumn{4}{|l|}{ Education, $N$ (percent) } \\
\hline Less than high school & $1722(13.66)$ & $1019(13.92)$ & $703(13.3)$ \\
\hline High school degree or GED & $6746(53.51)$ & $4140(56.57)$ & $2606(49.29)$ \\
\hline Some college & $798(6.33)$ & $485(6.63)$ & $313(5.92)$ \\
\hline College degree or higher & $3340(26.5)$ & 1675 (22.89) & 1665 (31.49) \\
\hline Years of parental education (range $0-17$ ), mean (SD) & $9.86(3.42)$ & $9.79(3.38)$ & $9.96(3.49)$ \\
\hline \multicolumn{4}{|l|}{ Depression before age $16, \mathrm{~N}$ (percent) } \\
\hline No & $12,261(97.26)$ & $7070(96.6)$ & $5191(98.18)$ \\
\hline Yes & $345(2.74)$ & $249(3.4)$ & $96(1.82)$ \\
\hline \multicolumn{4}{|l|}{ General health, $N$ (percent) } \\
\hline Excellent & $1714(13.6)$ & $984(13.44)$ & $730(13.81)$ \\
\hline Very good & $4181(33.17)$ & 2418 (33.04) & $1763(33.35)$ \\
\hline Good & $3914(31.05)$ & $2248(30.71)$ & $1666(31.51)$ \\
\hline Fair & $2128(16.88)$ & $1255(17.15)$ & $873(16.51)$ \\
\hline Poor & $669(5.31)$ & $414(5.66)$ & $255(4.82)$ \\
\hline Mean (SD) & $2.67(0.01)$ & $2.69(0.01)$ & $2.65(0.01)$ \\
\hline \multicolumn{4}{|l|}{ Physical abuse by either parent before age $18, N{\text { (percent })^{\mathrm{a}}}^{\mathrm{a}}$} \\
\hline No & $11,713(92.92)$ & $6747(92.18)$ & 4966 (93.93) \\
\hline Yes & $867(6.88)$ & $560(7.65)$ & $307(5.81)$ \\
\hline Missing & $26(0.21)$ & $12(0.16)$ & $14(0.26)$ \\
\hline Index of stressful life events in adulthood (range $0-5)$, mean (SD) ${ }^{b}$ & $0.33(0.68)$ & $0.32(0.66)$ & $0.35(0.71)$ \\
\hline Index of traumatic events in adulthood (range $0-7)$, mean $(S D)^{c}$ & $1.18(1.71)$ & $1.17(1.16)$ & $1.21(1.19)$ \\
\hline \multicolumn{4}{|l|}{ Demographics and characteristics } \\
\hline \multicolumn{4}{|l|}{ Marital status, $N$ (percent) } \\
\hline Married/partnered & $8868(70.35)$ & $4565(62.37)$ & $4303(81.39)$ \\
\hline Separated/divorced & $1523(12.08)$ & 1035 (14.14) & $488(9.23)$ \\
\hline
\end{tabular}


Table 1 (continued)

\begin{tabular}{|c|c|c|c|}
\hline & $\begin{array}{l}\text { Full sample } \\
(N=12,606)\end{array}$ & $\begin{array}{l}\text { Female } \\
(N=7,319)\end{array}$ & $\begin{array}{l}\text { Male } \\
(N=5,287)\end{array}$ \\
\hline Widowed & $1709(13.56)$ & $1429(19.52)$ & $280(5.3)$ \\
\hline Never married & $506(4.01)$ & $290(3.96)$ & $216(4.09)$ \\
\hline $\begin{array}{l}\text { Number of close relationships with children, friends and other family } \\
\text { members (range 0-221), mean (SD) }\end{array}$ & $10.03(10.07)$ & $10.17(0.11)$ & $9.84(10.85)$ \\
\hline Grand mean CESD-8, mean (SD) & $1.27(1.86)$ & $1.41(1.96)$ & $1.06(1.67)$ \\
\hline \multicolumn{4}{|l|}{ Predicted CESD-8 scale by age group $(\mathrm{CI})^{\mathrm{d}}$} \\
\hline $51-55$ years $* * *$ & $1.31(1.23,1.40)$ & $1.45(1.35,1.55)$ & $1.14(1.06,1.22)$ \\
\hline $56-60$ years $* * *$ & $1.27(1.20,1.34)$ & $1.40(1.32,1.49)$ & $1.11(1.04,1.18)$ \\
\hline $61-65$ years $* * *$ & $1.15(1.07,1.23)$ & $1.27(1.17,1.36)$ & $1.01(0.94,1.09)$ \\
\hline $66-70$ years $* * *$ & $1.15(1.09,1.21)$ & $1.26(1.19,1.34)$ & $1.01(0.95,1.07)$ \\
\hline $71-75$ years $* * *$ & $1.25(1.18,1.32)$ & $1.37(1.29,1.46)$ & $1.09(1.02,1.16)$ \\
\hline $76-80$ years $* * *$ & $1.37(1.28,1.46)$ & $1.53(1.42,1.64)$ & $1.20(1.11,1.28)$ \\
\hline $81-85$ years $* * *$ & $1.33(1.22,1.43)$ & $1.47(1.34,1.59)$ & $1.15(1.06,1.25)$ \\
\hline $86-90$ years $* * *$ & $1.43(1.28,1.59)$ & $1.58(1.40,1.75)$ & $1.23(1.10,1.36)$ \\
\hline $91-95$ years $* * *$ & $1.86(1.57,2.14)$ & $2.09(1.75,2.43)$ & $1.56(1.34,1.78)$ \\
\hline Severe depressive symptoms (CESD- $8>=4)$, percent & 8.37 & 9.98 & 6.06 \\
\hline \multicolumn{4}{|l|}{ Predicted percent of severe depressive symptoms $($ CESD- $8>=4)(C I)^{d}$} \\
\hline $51-55$ years $* * *$ & $9.72(8.60,10.85)$ & $11.34(10.00,12.67)$ & $7.70(6.61,8.79)$ \\
\hline $56-60$ years $* * *$ & $8.97(8.05,9.90)$ & $10.47(9.35,11.60)$ & $7.09(6.17,8.00)$ \\
\hline $61-65$ years $* * *$ & $7.46(6.45,8.46)$ & $8.72(7.55,9.89)$ & $5.87(4.91,6.82)$ \\
\hline $66-70$ years $* * *$ & $7.06(6.27,7.85)$ & $8.26(7.29,9.24)$ & $5.55(4.80,6.30)$ \\
\hline $71-75$ years $* * *$ & $7.51(6.59,8.43)$ & $8.78(7.65,9.91)$ & $5.91(5.06,6.75)$ \\
\hline $76-80$ years $* * *$ & $8.43(7.17,9.69)$ & $9.85(8.33,11.36)$ & $6.65(5.54,7.76)$ \\
\hline $81-85$ years $* * *$ & $7.89(6.45,9.33)$ & $9.22(7.52,10.92)$ & $6.21(4.96,7.46)$ \\
\hline $86-90$ years $* * *$ & $8.18(6.05,10.31)$ & $9.56(7.09,12.03)$ & $6.45(4.66,8.24)$ \\
\hline 91-95 years*** & $11.07(7.16,14.99)$ & $12.88(8.38,17.39)$ & $8.80(5.53,12.06)$ \\
\hline
\end{tabular}

$* * * P<0.001, * * P<0.01, * P<0.05$

Notes: Abbreviations: $S D$ standard deviation, $C I$ confidence interval

a Respondents were asked (1, yes; 2, no): "Before you were 18 years old, were you ever physically abused by either of your parents?" The number of missing for this physical abuse measure was scarce (only 12 cases out of 7,319 females; 14 cases out of 5,287 males) and they were not significantly different $(P>0.05)$ from the group who reported no physical abuse before age 18 . These cases were kept to maintain the same sample size that included the rest of the variables

${ }^{\mathrm{b}}$ Sum of ever experienced five stressful life events in the past 5 years as the respondent was asked:

(1) Have you involuntarily lost a job for reasons other than retirement at any point in the past 5 years?

(2) Have you been unemployed and looking for work for longer than 3 months at some point in the past 5 years?

(3) Was anyone else in your household unemployed and looking for work for longer than 3 months in the past five years?

(4) Have you moved to a worse residence or neighborhood in the past 5 years?

(5) Were you robbed or did you have your home burglarized in the past 5 years?

${ }^{\mathrm{c}}$ Sum of ever experienced seven traumatic events as respondents was asked

(1) Has a child of yours ever died?

(2) Have you ever been in a major fire, flood, earthquake, or other natural disaster?

(3) Have you ever fired a weapon in combat or been fired upon in combat?

(4) Has your spouse, partner, or child ever been addicted to drugs or alcohol?

(5) Were you the victim of a serious physical attack or assault in your life?

(6) Did you ever have a life-threatening illness or accident?

(7) Did your spouse or a child of yours ever have a life-threatening illness or accident?

${ }^{\mathrm{d}}$ Wald test results showed that gender differences are statistically significant at $P<0.001$ 